\title{
A possible disk mechanism for the 23d QPO in Mkn 501
}

\author{
J.H. Fan ${ }^{1}$, F.M. Rieger ${ }^{2}$, T.X. Hua ${ }^{1}$, U. C. Joshi ${ }^{1,3}$, \\ J. Li ${ }^{1}$, Y.X. Wang ${ }^{4}$, J.L. Zhou ${ }^{1}$, Y.H. Yuan ${ }^{1}$, J.B. Su${ }^{1}$, Y.W. Zhang ${ }^{1}$ \\ 1. Center for Astrophysics, Guangzhou University, Guangzhou 510400, China \\ 2. UCD School of Mathematical Sciences, University College Dublin, Belfield, \\ Dublin 4, Ireland \\ 3. Physical Research Laboratory, Ahmedabad 380 009, India \\ 4. College of Science and Trade, Guangzhou University, Guangzhou 511442
}

\begin{abstract}
Optically thin two-temperature accretion flows may be thermally and viscously stable, but acoustically unstable. Here we propose that the O-mode instability of a cooling-dominated optically thin two-temperature inner disk may explain the 23day quasi-periodic oscillation (QPO) period observed in the $\mathrm{TeV}$ and X-ray light curves of Mkn 501 during its 1997 high state. In our model the relativistic jet electrons Compton upscatter the disk soft X-ray photons to $\mathrm{TeV}$ energies, so that the instability-driven X-ray periodicity will lead to a corresponding quasi-periodicity in the $\mathrm{TeV}$ light curve and produce correlated variability. We analyse the dependence of the instability-driven quasi-periodicity on the mass $(\mathrm{M})$ of the central black hole, the accretion rate $(\dot{\mathrm{M}})$ and the viscous parameter $(\alpha)$ of the inner disk. We show that in the case of Mkn 501 the first two parameters are constrained by various observational results, so that for the instability occurring within a two-temperature disk where $\alpha=0.05-1.0$, the quasi-period is expected to lie within the range of 8 to 100 days, as indeed the case. In particular, for the observed 23-day QPO period our model implies a viscosity coefficient $\alpha \leq 0.28$, a sub-Eddington accretion rate $\dot{M} \simeq 0.02 \dot{M}_{\text {Edd }}$ and a transition radius to the outer standard disk of $r_{0} \sim 60 r_{g}$, and predicts a period variation $\delta P / P \sim 0.23$ due to the motion of the instability region.
\end{abstract}

Key words: Active Galactic Nuclei: Black Hole - Accretion Disk - Periodicity Individual: Mkn 501

$\overline{1}$ Corresponding author: J.H. Fan, email: fjh@gzhu.edu.cn

Preprint submitted to Elsevier

Received/Accepted 


\section{Introduction}

Strong variability is one of the common observational properties of $\mathrm{TeV}$ emitting Active Galactic Nuclei (AGNs) [10]. In many cases, the highly variable high-energy gamma-rays and the X-rays appear to be correlated with no time delays evident on day-scales, suggesting that the $\gamma$-rays may result from inverse Compton upscattering of lower energy photons. The first TeV blazar, for example, that was observed simultaneously in multiple bands from radio to TeV gamma-rays is Mrk 421. The first campaign, conducted in 1994 [39] on Mrk 421, revealed correlated variability between the keV X-ray and TeV gamma-ray emission. The gamma-ray flux varied by an order of magnitude on a timescale of 2 days and the X-ray flux was observed to double in $12 \mathrm{hr}$. On the other hand, the high-energy gamma-ray flux observed by EGRET, as well as the radio and UV fluxes, showed less variability than the $\mathrm{keV}$ or $\mathrm{TeV}$ bands. Another multiwavelength campaign on Mrk 421 performed in 1995 revealed another coincident $\mathrm{keV} / \mathrm{TeV}$ flare $[6,63$. The UV and optical bands also showed correlation during the flares. With the detection of $\mathrm{TeV}$ emission from Mrk 501 [52], several multiband campaigns were organized on Mrk 501 to verify whether such a behavior is a general property of TeV-emitting blazars or whether it is unique to Mrk 421.

The gamma-ray blazar Mkn 501, detected as a strong TeV emitter in 1995 [52], is one of the closest $(z=0.0337)$ and brightest BL Lacertae objects. Historically (i.e., prior to 1997), its spectral energy distribution (SED) $\nu F_{\nu}$ resembles that of X-ray selected BL Lac objects, having a peak in the extreme UV-soft X-ray energy band [24,57]. Earlier optical observations of Mkn 501 have shown variations of up to $1 .{ }^{m} 3$ and polarized emission up to $P_{\text {opt }} \simeq 7 \%$ [20].

During its active state in 1997, where Mkn 501 was monitored in the 2-10 keV X-ray band by the all sky monitor(ASM) on board RXTE and in the TeV energy band by several Cherenkov telescope groups [1,9,17,32,51,56], both Xrays and $\mathrm{TeV}$ gamma-rays increased by more than 1 order of magnitude from quiescent level [9,48]. Analysis of the X-ray and $\mathrm{TeV}$ data showed, that the variations were strongly correlated between both bands, yet only marginally correlated with the optical UV band [9, 17,32,47]. While the synchrotron emission peaked below $0.1 \mathrm{keV}$ in the quiescent state, in 1997 it peaked at $100 \mathrm{keV}$. This is the largest shift ever observed for a blazar [48]. Earlier investigations of the 1997 April flare in Mkn 501 [48], showed that its origin may be related

to a variation of $\gamma_{\max }$ together with an increased luminosity and a flattening of the injected electron distribution.

During the 1997 high state, the X-ray and TeV light curves displayed a quasiperiodic signature [22,30,51]. A detailed periodicity analysis, based on the 
TeV measurements obtained with all Cherenkov Telescopes of the HEGRACollaboration and the X-ray data with RXTE, was performed by Kranich et al. [30,31] and more recently also by Osone [45] including Utah TA data. The results indeed strongly suggest the existence of a 23 day periodicity, with a combined probability of $p \simeq 3 \times 10^{-4}$ in both the $\mathrm{TeV}$ and the $\mathrm{X}$-ray light curves covering the same observational period [30131. 2 Rieger and Mannheim (2000) have shown that the origin of these QPOs may be related to the presence of a binary black hole in the center of Mkn 501. While this may well be possible, we explore here an alternative scenario, where the observed QPOs are related to accretion disk instabilities.

In a seminal paper, Shapiro et al. (1976) (SLE) have pointed out that a hot (Compton cooling-dominated) optically thin two-temperature accretion disk may be present in the inner region of the standard optically thick disk [59], whenever the SLE inequality $\alpha^{1 / 4} \dot{M}_{*}^{2} M_{*}^{-7 / 4} \geq 0.6$ is satisfied, where $\dot{M}_{*}=\dot{M} /\left(10^{17} \mathrm{gs}^{-1}\right), M_{*}=M /\left(3 M_{\odot}\right), \dot{M}$ is the accretion rate of the disk, $M$ the mass of the central black hole, and $\alpha$ the viscosity parameter, constrained by the model to lie within the range $0.05<\alpha<1$. Later work 49,50, has shown that the SLE configuration might be thermally unstable (although less than the standard disk) if the simple standard viscosity prescription is employed. However, relatively small changes in the viscosity law can already ensure a stable configuration [49], in particular, if stabilizing effects of a disk wind are fully taken into account. A kind of SLE two-temperature disk structure may thus well exist in the inner region of BL Lac type objects [66,67,8] and provide a possible explanation for the $\mathrm{X}$-ray and $\mathrm{TeV}$ variability phenomenon in Mkn 501. For, firstly, Compton processes in a inner two-temperature disk with electron temperature $T_{e}$ of about $10^{9} \mathrm{~K}$ (and ion temperature one or two orders of magnitude higher) can produce (steep) X-ray power-law spectra, in contrast to the standard disk model that can only produce emission up to the optical-UV band [58,66]. Secondly, analysis of the linear stability of an optically thin two-temperature disk around a compact object shows that the disk is subject to a radial pulsational instability (inertial-acoustic mode instability) [69. This possible mode of pulsational overstability, in which radial disk oscillations with local Keplerian frequencies become unstable against axisymmetric perturbations, occurs if the viscosity coefficient increases sufficiently upon compression [26]. In this case, thermal energy generation due to viscous dissipation increases during compression, leading to amplification of the oscillations analogous to the role played by nuclear energy generation in stellar pulsations. As we demonstrate below, the occurrence of such a type of disk oscillation may well account for some quasi-periodic time variability in AGNs in a way similar to Galactic black hole candidates [5,23,40,70].

$\overline{2}$ Note that no QPOs have been seen by MAGIC during 1998-2000, when the source was not very active [3], suggesting that the QPOs only occur during a very active stage. 


\section{$2 \quad$ Model and Results}

\subsection{Model}

Due to the Lightman \& Eardley secular instability [36,37] of the standard geometrically thin, optically thick disk model [59], a two-temperature disk configuration is likely to be present in the inner region of the standard disk. As shown by Shapiro et al. [58], the outer boundary of such a two-temperature disk is determined by

$$
r_{0 *}^{21 / 8} \zeta^{-2}\left(r_{0}\right) \approx 3.85 \times 10^{6} \alpha^{1 / 4} M_{7}^{-7 / 4} \dot{M}_{24}^{2}
$$

where $r_{0}=r_{0 *} \frac{G M}{c^{2}}$ is the radius of the outer boundary, $M_{7}$ is the mass of the central black hole in units of $10^{7} M_{\odot}, \dot{M}_{24}$ is the accretion rate in units of $10^{24}$ $\mathrm{g} \mathrm{s}^{-1}=0.016 M_{\odot} / \mathrm{yr}, \alpha$ is the viscosity parameter constrained to be within the range of 0.05 to 1.0 , and $\zeta=1-\left(\frac{r_{0 *}}{6}\right)^{-1 / 2}$ expresses the boundary condition that the viscous stress must vanish at the inner edge of the disk.

The total luminosity of a two-temperature disk, integrated from the inner edge to $r_{0}$, is of order

$$
L_{r 0,44}=\frac{3}{4}\left[1+2\left(\frac{1}{6} r_{0 *}\right)^{-3 / 2}-18 / r_{0 *}\right] \dot{M}_{24}
$$

where $L_{r 0,44}$ is in units of $10^{44} \mathrm{erg} \mathrm{s}^{-1}$. Combining relations (1) and (2), one obtains

$$
\left[1+2\left(\frac{r_{0 *}}{6}\right)^{-3 / 2}-\frac{18}{r_{0 *}}\right] r_{0 *}^{21 / 16}\left[1-\left(\frac{r_{0 *}}{6}\right)^{-1 / 2}\right]^{-1}=2.62 \times 10^{3} L_{r 0,44} \lambda
$$

where $\lambda \equiv\left(\alpha M_{7}^{-7}\right)^{1 / 8}$. Obviously, since $\lambda$ is relatively insensitive to $\alpha$, the outer radius $r_{0 *}$ and the accretion rate $\dot{M}$ can be uniquely determined, once the central black mass $M$ and the observed radiative output $L_{r 0,44}$ are known.

Following Shapiro et al. [58] (see also [66]) we assume, that a hot (coolingdominated) optically thin two-temperature region forms within the inner region of the standard disk. Hence, the classical outer and middle regions of the standard Shakura \& Sunyaev (SS) disk model [59] describe the outer portions of our disk model. The inner region of the cool SS disk, whose outer boundary lies at the point where the radiation pressure $P_{R}$ matches the gas pressure $P_{G}$, extends inwards to the radius $r_{0}$, where $P_{R}=3 P_{G}$. The radius $r_{0}$ marks the outer boundary of the two-temperature inner region, which then extends inward to the innermost radius $3 r_{g}$ for a non-rotating black hole. In short, 
the assumed disk configuration is assumed to be identical to the standard SS model for $r \geq r_{0}$, but described by the two-temperature structure equations for $r<r_{0}$. The disk thickness, the density, and the ratio $\frac{h}{r}$ for the equilibrium, two-temperature inner disk then satisfy the following equations:

$$
\begin{aligned}
h & =5.29 \times 10^{11} M_{7}^{7 / 12} \dot{M}_{24}^{5 / 12} \alpha^{-7 / 12} \zeta^{5 / 12} r_{*}^{7 / 8} \mathrm{~cm}, \\
\rho & =1.14 \times 10^{-11} M_{7}^{-3 / 4} \dot{M}_{24}^{-1 / 4} \alpha^{3 / 4} \zeta^{-1 / 4} r_{*}^{-9 / 8} \mathrm{~g} \mathrm{~cm}^{-3} \\
h / r & =0.316 \times \mathrm{M}_{7}^{-5 / 12} \dot{\mathrm{M}}_{24}^{5 / 12} \alpha^{-7 / 12} \zeta^{5 / 12} \mathrm{r}_{*}^{-1 / 8} .
\end{aligned}
$$

Typically, the emission spectrum of a hot $\left(T_{e} \sim 10^{9} \mathrm{~K}\right)$ inner disk is bremstrahlung unless a soft photon source is present, in which case the spectrum may become a power-law. In our hybrid model a fraction of the optical-UV soft photons from the outer SS disk is assumed to be intercepted by the hot SLE inner disk and Comptonized to form the X-ray power-law spectrum, similar as in 58,66. If Comptonization is not saturated, as possible in the presence of a copious soft photon source (such as a cold SS component), the soft photons will be upscattered into a power-law distribution over some energy range. The resulting disk spectrum above the input soft energy $E_{s}=h \nu_{s}$ is approximately of the form

$$
F_{\nu} \propto \nu^{-\alpha_{x}} \exp \left(-h \nu / k T_{e}\right)
$$

where $\alpha_{x}=\left(2.25+4 /\left[y\left(1+f_{T}\right)\right]\right)^{1 / 2}-3 / 2>0, f_{T}=2.5 \theta+1.875 \theta^{2}(1-\theta)$, $\theta \equiv k T_{e} / m_{e} c^{2}$ and $y \sim 1$ for unsaturated Compton cooling, i.e., the spectrum resembles a power-law with index $\alpha_{x}$ for $E>E_{S}$ and $E \ll k T_{e}$, and shows an exponential cut-off for $E \gtrsim k T_{e}$ [58,66]. The corresponding SLE disk SED $\nu F_{\nu}$ peaks at $E_{p}=\left(1-\alpha_{x}\right) k T_{e}$, i.e., usually at around some tens of $\mathrm{keV}$, with $E_{p}$ being higher for smaller indices. In particular, for $k T_{e}=200 \mathrm{keV}$ $\left(T_{e}=2.3 \times 10^{9} \mathrm{~K}\right)$ one may have $\alpha_{x} \simeq(2.25+1.85 / y)-1.5 \in[0.4,0.8]$ for $y \in[0.6,1.3]$.

As first shown by Kato (1978), accretion disks can undergo (radial-azimuthal) pulsational instabilities and thereby cause quasi-periodic variability. If a fluid element in the disk is perturbed from its equilibrium position, it oscillates in the radial direction with the epicyclic frequency, as the difference between gravitational and inertial (Coriolis and centrifugal) force appears as a restoring force. Due to this property, axisymmetric perturbations can propagate in the disk as inertial acoustic waves. As shown by Kato, a viscous disk can be pulsationally unstable against this type of oscillations under certain conditions: By studying the stability properties of an optically thin disk to axisymmetric, local (wavelength $\lambda \ll$ radial size of disk) and nearly radial $\left(v_{r} \gg v_{z}\right)$ oscillations, he found that the disk becomes unstable if the coefficient of the viscosity increases sufficiently rapidly with density and temperature, and that 
the frequency of oscillations essentially corresponds to the angular frequency $\Omega$ of the disk, independent of wavelength. More recently, Wu (1997) has studied in detail the radial pulsation instability of optically thin two-temperature accretion disks, demonstrating that in a geometrically thin, cooling-dominated two-temperature disk the acoustic O-mode (outward-propagating) is always unstable. It has been argued, that pulsational overstability might explain some of the QPO phenomena observed in cataclysmic variables (CVs) and Galactic black hole systems [5,11,40,43], and be important for a proper understanding of periodic variability in AGNs [23]. As we show in the the next section, Mkn 501 may indeed represent a promising AGN candidate, where pulsational overstability becomes apparent.

Numerical simulations of pulsational instability of geometrically thin, optically thick (one-temperature) $\alpha$ disks show that inertial acoustic waves are excited and propagate both inwards and outwards periodically, immediately growing to shock waves and resulting in a time-varying local accretion rate exceeding the imposed input value $\dot{M}$ by one or two orders of magnitude [23,43]. Global nonlinear calculations of such systems indeed demonstrate that (i) the net mass flow changes sign by a significant amount according to the direction of the oscillatory flow, although the disk seems to maintain its ability to transport on average the initially imposed $\dot{M}$ [27], and that (ii) the oscillations also induce small relative changes in other variables (e.g., temperature or surface density) [43. In particular, the luminosity variations caused by overstable oscillations in such systems are expected to be (only) at the percent level, even if local variables such a mass accretion rate and radial velocity change significantly 11,27,43. Unfortunately, to our knowledge, no simulation of pulsational overstability for a SLE type, optically thin, cooling-dominated two-temperature disk configuration has been performed up to now. This makes it difficult to draw solid (quantitative) conclusions for the SLE case as the simulations performed so far consider physical environments quite different from that of a hot, optically thin, gas-pressure supported two-temperature accretion disk. Global simulations of optically thin, two-temperature disk configurations are certainly required to remedy this problem. Nevertheless, it seems very likely that significant changes in local accretion rate during an oscillatory period represent a genuine qualitative feature associated with pulsational overstability. If so, then substantial quasi-periodic changes in the total disk luminosity might be expected for the SLE case (cf. [58], eqs.[1] and [21]).

In principle, the observable period of the pulsational instability in a viscous disk is of order [70]

$$
\begin{aligned}
P & \approx(1+z) \alpha^{-1} \Omega^{-1} \\
& =5.78 \times 10^{-4}(1+z) M_{7} r_{*}^{3 / 2} \alpha^{-1}[\text { days }]
\end{aligned}
$$


where $\Omega^{-1}=\sqrt{r^{3} / G M}$ is the local Keplerian timescale, $r_{*}$, in units of $\frac{G M}{c^{2}}$, is the radius at which the pulsation instability occurs, and $z$ is the redshift of the source. Obviously, since the disk structure depends significantly on the radius, the period of the pulsational instability is different from one radius to another. Yet, by using eq.(3) we can calculate the radius $r_{0}$, at which the disk becomes unstable to radial pulsation for a given luminosity, and so derive an upper limit on the range of possible periods. In fact, these upper limits may offer a very useful guide, since numerical disk simulations suggest that the oscillations may always be trapped near the boundaries of the discs [27, 46].

\subsection{Application to Mkn 501}

The $\gamma$-ray blazar Mkn 501 was the second source after Mkn 421 to be detected at TeV energies in 1995 by the Whipple observatory [52]. In 1997 the source came into much attention when it went into a remarkable state of strong and continuous flaring activity, becoming the brightest source in the sky at TeV energies [1,2,9,17,22,51]. BeppoSAX observations during a strong outburst in April 1997 showed, that the spectrum was exceptionally hard ( $\alpha \leq 1$, $\left.F_{\nu} \propto \nu^{-\alpha}\right)$ over the range $0.1-200 \mathrm{keV}$, indicating that the X-ray power output peaked at $100 \mathrm{keV}$ or higher energies [48]. These observations implied that the peak frequency has moved up by more than two orders of magnitude (persistent over a timescale of $\sim 10 \mathrm{~d}$ ) and that the (apparent) bolometric luminosity of Mkn 501 has increased by a factor of $\gtrsim 20$ compared to previous epochs 24,32, 48,57,64. Optical observations on the other hand indicated, that the source was still relatively normal and thus suggested that the variations are confined to energies above $\sim 0.1 \mathrm{keV}$ [47]. Further observations with BeppoSAX in April-May 1998 and May 1999 during periods of lower TeV flux showed that the peak frequency has decreased to $\sim 20$ and $\sim 0.5 \mathrm{keV}$, respectively [64.

A comprehensive analysis of the temporal characteristics of the gamma-ray emission from Mkn 501 in 1997 showed that the TeV emission varied significantly on time scales of between (5-15) hours [1]. If this short time variability is via inverse Compton processes related to accretion disk phenomena (see $\S 3$ ), we can estimate an upper limit for the black hole mass in Mkn 501 by taking the variability timescale to be of order of the Keplerian orbital period at the innermost stable orbit $r_{\mathrm{ms}}$, suggesting

$$
M_{7} \leq 7.9\left(\frac{\Delta t}{10 \mathrm{hr}}\right) \frac{1}{(1+z)}
$$

for a non-rotating black hole where $r_{\mathrm{ms}}=3 r_{g}=\frac{6 G M}{c^{2}}$, and thus a black hole mass of $M \leq(3.8-11.5) \times 10^{7} M_{\odot}$ for the (5-15) hour timescale observed. Note, 
that this mass upper limit may be somewhat higher, if a pseudo-Newtonian potential is employed, in which case one may find $M \leq(5.7-17.2) \times 10^{7} M_{\odot}$. Eq. (9) essentially assumes that the observed short-time variability is dominated by processes occurring close to the innermost stable orbit. This seems justified as long as the associated timescale $\left(t \sim 3.6 \times 10^{4} \mathrm{sec}\right.$ in the lab. frame) is larger than (i) the transverse light crossing time of the source as measured in the lab. frame, i.e., $t_{l} \sim r_{b} /\left[\Gamma_{b} c\right]<t$, where $r_{b} \sim z / \Gamma_{b}$ with $\Gamma_{b} \sim 15$ the bulk Lorentz factor and $z \sim 5 \times 10^{2} r_{g}$ the distance from the central engine, cf. [15], and (ii) the characteristic shock acceleration timescale $t_{\text {acc }} \lesssim 10^{3} r_{\text {gyr }} /\left(\Gamma_{b} c\right)<t_{l}$ [55], where $r_{\text {gyr }}$ is the electron gyroradius for intrinsic magnetic field strengths $\gtrsim 0.01 \mathrm{G}$ and comoving electron Lorentz factors of $\sim 10^{5}$ (see below), which seems indeed to be the case. Based on the above noted, as well as related considerations in [54], we adopt a black hole mass of $M=9 \times 10^{7} M_{\odot}$, for which $r_{g} \simeq 2.66 \times 10^{13} \mathrm{~cm}$, as fiducial value in our calculations below.

Detailed observations of Mkn 501 during the 1997 active phase showed that its X-ray emission was highly variable, although the soft X-ray flux (up to a few keV) did not change dramatically 32,34,41,48. BeppoSAX observations in 1997 give $(2-10) \mathrm{keV}$ fluxes in the range $(1.69-5.24) \times 10^{-10} \mathrm{erg} \mathrm{cm}^{-2}$ $\mathrm{s}^{-1}$, with an average value of $2.50 \times 10^{-10} \mathrm{erg} \mathrm{cm}^{-2} \mathrm{~s}^{-1}$ [41], corresponding to a mean (bolometric) luminosity of $\left\langle L_{X}\right\rangle=6.2 \times 10^{44} \mathrm{erg} \mathrm{s}^{-1}$, assuming $\mathrm{H}_{0}=72 \mathrm{~km} / \mathrm{s} / \mathrm{Mpc}$ and a flat Universe with $\Omega_{M}=0.27$. If the observed periodicity in the (2-10) $\mathrm{keV}$ band is caused by a pulsational disk instability, a non-negligible fraction $f<1$ of this X-ray luminosity has to be produced by the inner disk, the other part being provided by the relativistic jet. Each surface of the disk then produces a characteristic (2-10) keV luminosity of $L_{2-10 \mathrm{keV}}=3.1 \times 10^{44} \mathrm{f} \mathrm{erg} \mathrm{s}^{-1}$. Simulations of pulsational instability discussed at the end of $\S 2.1$ indicate that the oscillations are accompanied by periodic, large amplitude variations in local accretion rate (exceeding the average $\dot{M}$ by over an order of magnitude) and possibly trapped near the outer edge of the disk. Simple quantitative modelling then suggests that the total luminosity of a SLE type disk may vary by up to a factor of a some few, i.e., $f$ may be as small as $\sim 0.1$. Using the observed soft X-ray power-law index $\alpha_{x} \simeq 0.7$ [33,41,47], we may estimate the observationally required, total integrated SLE disk luminosity $L_{r 0}$ by calculating the ratio $\eta \equiv L_{2-10 \mathrm{keV}} / L_{r 0}$ of the soft X-ray to total disk luminosity from the emitted disk spectrum (see eq. 77), which for $k T_{e} \simeq 200 \mathrm{keV}$ gives $\eta \simeq 1 / 5$. This suggests that the total (average) SLE

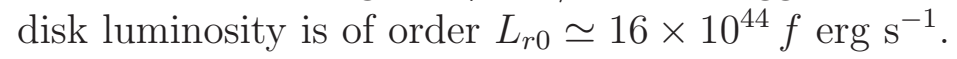

As shown below, low $f$ values are more preferable in the hybrid SLE model and we will thus henceforth adopt a total SLE disk luminosity $<L_{r 0}>=1.75 \times 10^{44}$ $\operatorname{erg~s}^{-1}(f=0.11)$ for explicit calculation (see however also Fig. 1 for the more general case). Given the constraints on the viscous parameter $\alpha$ and using $M=9.0 \times 10^{7} \mathrm{M}_{\odot}$, we may then obtain the following results for the transition 
radius $\mathrm{r}_{0 *}$, the maximum pulsational period $\mathrm{P}$ and the ratio $\frac{h}{r}$, namely, for $\alpha=0.05$ we have: $\mathrm{r}_{0 *}=97, \mathrm{P}=100$ days, $\frac{h}{r_{0}}=0.55$; while for $\alpha=1.0$ one finds: $\mathrm{r}_{0 *}=130, \mathrm{P}=8$ days, $\frac{h}{r_{0}}=0.09$, respectively. In both cases the accretion rate is similar, i.e., $\dot{M}_{24} \simeq 2.7$, corresponding to $\dot{M} \simeq 0.02 \dot{M}_{\text {Edd }}$, and consistent with the condition (see $\S 1$ ) for a SLE configuraton to exist. We note that for BL Lac objects the transition radius between an inner optically thin two-temperature and an outer standard disk has been estimated to lie within the range $r_{0 *} \simeq(40-150)$ for $\dot{M} / \dot{M}_{\text {Edd }}=0.01$ [8], which seems well consistent with the values derived above. Based on these results, we can also roughly estimate the luminosity $L_{s s} \sim 2 \pi r^{2} \sigma T^{4}$ associated with the SS disk component at $r \geq r_{0}$ : Employing the thin cold disk temperature relation $T \simeq 6.3 \times 10^{5} \dot{m}^{1 / 4}\left(10^{8} M_{\odot} / M\right)^{1 / 4}\left(r_{g} / r\right)^{3 / 4} \simeq 1.3 \times 10^{4} \mathrm{~K}$ with $r=r_{0} \simeq 50 r_{g}$ one finds that $L_{s s} \simeq 1.8 \times 10^{43} \mathrm{erg} / \mathrm{s}$, and that the emission is maximized at frequencies around $f \simeq 3 k T / h \simeq 8 \times 10^{14} \mathrm{~Hz}$. These findings imply a Compton energy enhancement factor $A$ considerably larger than $L_{r 0} / L_{s s} \simeq 10$.

Spectroscopic data for Mkn 501 suggest, that the normal bolometric (photoionization-equivalent) luminosity $L_{b}$ of the disk component driving the NLR photoionization is of order a few times $10^{43} \mathrm{erg} / \mathrm{s}$, corresponding to a photon number per second that can ionize hydrogen of $N_{b}(H) \lesssim L_{b} /\left(h \nu_{1}\right) \sim 10^{54} \mathrm{~s}^{-1}$, where $\nu_{1}=13.6 \mathrm{eV} / h$. Here, $L_{b}$ has been estimated based on the $L_{b}-L(H \alpha)$ relation, i.e., $\log L_{b}=1.176 \log L(H \alpha)-4.91$ [35], with an observed (mean) narrow $\mathrm{H} \alpha$ line luminosity for $\mathrm{Mkn} 501$ of $\simeq 10^{41} \mathrm{erg} / \mathrm{s}$ [61, which gives $L_{b} \sim$ $2 \times 10^{43} \mathrm{erg} / \mathrm{s}$ (with substantial scatter). We note that this value is very close to the one estimated by Barth et al. (2002) using emission line measurements for four nearby FR I radio galaxies. Given the properties of the cold SS disk component derived above, the main part of the ionizing photons per second is expected to come from the SLE component (unless its existence is only coupled to active source stages). The number of photons emitted by the SLE inner disk per second, which can ionize hydrogen is given by $N(H)=\int_{\nu_{1}}^{\infty} L_{\nu} /[h \nu] d \nu$, where $L_{\nu}$ is the specific SLE luminosity (cf. eq. [7). Using the values adopted above, i.e., $\alpha_{x} \simeq 0.7, k T_{e} \simeq 200 \mathrm{keV}$ and $L_{2-10 \mathrm{keV}}=5.8 \times 10^{44} \rho f \mathrm{erg} \mathrm{s}^{-1}$ ( $\rho \lesssim 0.3$ and $\rho=1$ for the quiescent and active source stage, respectively, cf. [41]), we can find $N(H) \simeq 4.5 \times 10^{55} \rho \mathrm{f} \mathrm{s}^{-1}$, which for $\rho \simeq 0.2$ and $f \simeq 0.1$ is comparable to $N_{b}(H)$, and may thus qualify the choice of $f$ above.

As mentioned in $\S 1$, detailed periodicity analysis of the X-ray and TeV lightcurves of Mkn 501 during its high state in 1997 has provided strong evidence for a 23-day period in both energy bands [30,31,45]. As shown above, such a period falls within the range of possible pulsational periods, suggesting that it may be well explained by the pulsational instability of a two-temperature SLE disk. In particular, by using $P=23$ day and eqs. (3) and (7), we can place an upper limit on the allowed viscosity parameter and the expected transition radius. For the characteristic values derived above, one obtains $\alpha \leq$ 


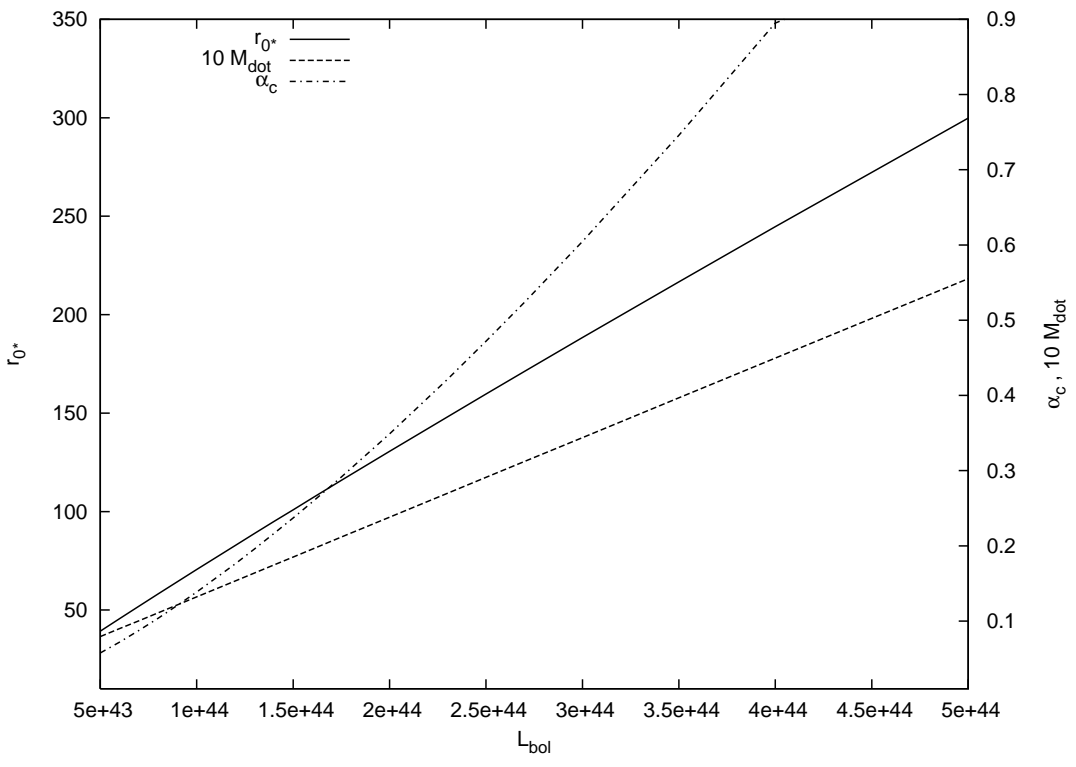

Fig. 1. Outer SLE radius $r_{0 *}$, accretion rate $M_{\text {dot }} \equiv \dot{M} / \dot{M}_{\text {Edd }}$ and upper limit for the viscosity coefficient $\alpha \leq \alpha_{c}$ as a function of the luminosity of a two-temperature SLE disk. Parameters $M=9 \times 10^{7} M_{\odot}$ and $P_{\text {obs }}=23 \mathrm{~d}$ have been employed in the calculations.

0.28 , corresponding to $\mathrm{r}_{0 *}=115$ and $\frac{h}{r_{0}} \sim 0.20$. The more general case is shown in Fig. (1), where the dependency of these results on the total SLE disk luminosity has been calculated.

The changing SLE disk luminosity will lead to a variable seed photon field for Compton upscattering to the high energy regime by the nonthermal particles in the relativistic blazar jet. The disk radiation entering a moving plasma blob from behind appears de-boosted by a factor $\sim \Gamma_{b}^{2}$ in the comoving frame of the outflowing plasma. It will nevertheless dominate over possible (quasi-isotropic) disk photons that are scattered by diffusive gas or clouds into the direction of the source, provided the distances $z$ to the central engine is smaller than

$$
z \lesssim 7 \times 10^{3}\left(\frac{15}{\Gamma_{b}}\right)^{2}\left(\frac{R_{\mathrm{sc}}}{1 \mathrm{pc}}\right)\left(\frac{0.001}{\tau_{\mathrm{sc}}}\right)^{1 / 2} r_{g}
$$

where $R_{\mathrm{sc}}$ is the distance and $\tau_{\mathrm{sc}}$ the mean scattering depth of the electron scattering cloud [15]. This usally places the emission site at distances between hundred (to avoid $\gamma \gamma$ absorption) and some thousand $r_{g}$.

As shown by Dermer and Schlickeiser (1993), scattering of X-ray $(\gtrsim 0.1 \mathrm{keV})$ photons entering from behind will generally take place in both the Thomson and the Klein-Nishina regime for a large range of (lab. frame) soft photon energies $\epsilon_{\mathrm{ph}}$, i.e., for energies in the range $\Gamma_{b} m_{e} c^{2} / \gamma<\epsilon_{\mathrm{ph}}<4 \Gamma_{b} \gamma m_{e} c^{2}$, where $\Gamma_{b} \sim 15$ is the jet bulk Lorentz factor. An full treatment of the scattering process is thus a complicated matter and beyond the scope of the paper. However, 
we can still get some order-of-magnitude insight into the associated photon luminosity by approximating the scattering process by its Thomson limit: The maximum value of the scattered photon energy $\epsilon_{\mathrm{ph}}^{s}$ in the lab. frame is given by $\epsilon_{\mathrm{ph}}^{s} \simeq 0.5 \gamma^{2} \epsilon_{\mathrm{ph}}[13]$. Upscattering of soft X-ray photons $(\gtrsim 0.2 \mathrm{keV})$ to the $\mathrm{TeV}$ regime thus requires comoving electron Lorentz factors of $\gamma \sim 10^{5}$. Scattering will always take place in the Thomson regime for (lab.frame) soft photon energies $\epsilon_{\mathrm{ph}}<\Gamma_{b} m_{e} c^{2} / \gamma \lesssim 0.08 \mathrm{keV}$ [14], suggesting that our approximation is indeed not too bad if we take an analogous reduction of the Thomson crosssection by a factor of two or so into account. For photons entering from behind the single inverse Compton power in the comoving blob frame for scattering by a relativistic electron with Lorentz factor $\gamma$ then becomes, cf. [15],

$$
P_{\mathrm{Comp}} \sim \frac{c}{6} \sigma_{\mathrm{T}} \frac{\gamma^{2}}{\Gamma_{b}^{2}} \frac{L_{\mathrm{r} 0}}{2 \pi z^{2} c}
$$

Suppose, that electrons are continuously accelerated at a shock front into a characteristic non-thermal (isotropic) differential power law particle distribution in the comoving frame of $n(\gamma)=n_{0} \gamma^{-2}$ for $10^{2} \lesssim \gamma_{1} \leq \gamma \leq \gamma_{\max }$ with $\gamma_{\max } \sim 2 \times 10^{6}$ roughly given by the balance between acceleration and external Compton cooling for $B \sim 0.05 \mathrm{G}$ and $z \sim 500 r_{g}$. Due to $\gamma_{\max } \propto L_{r 0}^{-1 / 2}$, an increase of the SLE disk field will lead to a decrease in the maximum Lorentz factor. As particle are advected downstream and no longer efficiently accelerated, the distribution steepens to $n(\gamma) \propto \gamma^{-3}$ for momenta where particles have had sufficiently time to cool, resulting into a broken power law electron distribution with a break energy of $\gamma_{b}$. We may estimate $\gamma_{b}$ by setting the (comoving) external Compton cooling time scale equal to the (comoving) dynamical time scale $\sim z /\left(\Gamma_{b} c\right)$, which gives $\gamma_{b} \sim 2 \times 10^{5}$. For typical values $n_{0} \gamma_{1} \sim 10^{4} \mathrm{~cm}^{-3}$, e.g., [47,33], the associated observable Compton VHE luminosity can thus be estimated from $L_{\text {obs }} \sim \Gamma_{b}^{4} P_{\text {Comp }} N_{\text {eq }} \Delta V$ where $N_{\mathrm{b}} \sim n\left(\gamma_{\mathrm{b}}\right) \gamma_{\mathrm{b}}$ and $\Delta V \sim 4 \pi\left(z / \Gamma_{b}\right)^{3} \Gamma_{b}$ is the volume scale of the emitting blob measured in the comoving frame. This gives a Compton luminosity

$$
L_{\mathrm{TeV}} \sim L_{\mathrm{r} 0}\left(\frac{z}{500 r_{g}}\right)\left(\frac{\gamma_{\mathrm{b}}}{10^{5}}\right)\left(\frac{N \gamma_{1}}{10^{4} \mathrm{~cm}^{-3}}\right),
$$

which is of the order of the observed VHE luminosity above $1.5 \mathrm{TeV}$ during the 1997 high state [1,47]. This suggests that changes in the disk photon field driven by a pulsational instability may indeed be responsible for the observed modulation of the TeV emission. During the quiescent state on the other hand, the X-ray flux is typically about ten times smaller (cf. §2.2). Hence, even if a SLE type disk is present in the quiescent state (see however below), the expected TeV contribution due to inverse Compton scattering of disk photon is much smaller, perhaps even swamped by synchrotron self-Compton emission. The 1997 observations of Mkn 501 showed that the observed TeV spectrum 
gradually steepened with energy starting at around $3 \mathrm{TeV}$, approaching a logarithmic spectral $\left(\nu F_{\nu}\right)$ slope above $5 \mathrm{TeV}$ of $\simeq 1.7 \pm 0.33 \pm 0.6$ including systematical and statistical errors [2,28]. However, as shown for example by Konopelko et al. (2003), this curvature at around $3 \mathrm{TeV}$ is likely to be caused by gamma-ray absorption in the intergalactic infrared background field, suggesting that the peak in the intrinsic (de-absorped) spectrum due to increasing Klein-Nishina effects is around $8 \mathrm{TeV}$. In principle, it appears possible that due to the strong angle dependence of the scattered flux on the angle of the impinging soft photons, photons from the outer SS disk part, i.e. from disk radii $\sim z$, could make an important inverse Compton contribution as well. Redoing the calculations presented in [15] for lab.frame angles of the incident photons of $\sim \pi / 4$ gives a total photon energy density of the soft photons in the comoving frame of $u_{\mathrm{ph}}^{s} \simeq\left(1-\beta_{b} / \sqrt{2}\right)^{2} u_{\mathrm{ph}, \mathrm{s}}^{\star}$ where $u_{\mathrm{ph}, \mathrm{s}}^{\star} \simeq L_{\mathrm{SS}}(r 0 / z) /\left(4 \pi z^{2} c\right)$ is the corresponding energy density in the lab. frame. As the SLE contribution $u_{\mathrm{ph}}^{\mathrm{SLE}}$, entering from behind, appears de-boosted by a factor of $\simeq 4 \Gamma_{b}^{2}$ [15] compared to its lab. frame value, the ratio of comoving energy densities becomes

$$
\frac{u_{\mathrm{ph}}^{s}}{u_{\mathrm{ph}}^{\mathrm{SLE}}} \sim 0.35\left(\frac{L_{\mathrm{r} 0}}{L_{\mathrm{SS}}}\right)\left(\frac{z}{r_{0}}\right),
$$

using $\Gamma_{b}=15, L_{\mathrm{r} 0} / L_{\mathrm{SS}} \sim 10$ and $z / r_{0} \sim 10$, indicating that the SLE part dominates during the high state even if we assume that the SS disk is not truncated at radii of $\sim 500 r_{g}$. Note however, that the latter may well be the case if a binary black hole system exists in the center of Mkn 501 as argued for by several authors (see discussion).

\section{Discussion}

The X-ray to $\mathrm{TeV}$ spectra of $\mathrm{TeV}$ blazars have been often interpreted within a one-zone SSC model, e.g. [4, 16, 42,62,64]. However, if a two-temperature disk structure is present in some of these sources, the real situation may be more complex as the X-ray radiation from a two-temperature disk, for example, may represent an additional, non-negligible source of seed photons for the inverse Compton scattering to $\mathrm{TeV}$ energies, in particular during active source stages, likely to be associated with changes in accretion history. Hence our model assumes, that a pulsational instability occurring within a two-temperature disk leads to observable, periodic variations of its X-ray radiation field. Part of this periodically modulated X-ray emission will enter the jet and (in addition to direct synchrotron photons) serves as seed photons for Compton-upscattering to $\mathrm{TeV}$ energies similar as in [13,14. Our model thus takes it that both, a direct synchrotron self Compton and an external Compton contribution are relevant 
for modelling the SED of Mkn 501, the seed photons for external Comptonupscattering consisting of both, the infrared-optical seed photons from the (quasi-steady) SS disk component and the variable X-ray photons from the two-temperature disk component. A related, but more simpler scenario, assuming the seed photons for inverse Compton scattering to be provided by a (direct) synchrotron plus an quasi-steady flux component comparable to the observed infrared-optical flux, has been proposed by Pian et al. (1998) in order to account for the different degrees of SED variations of Mkn 501 at X-ray and sub-X-ray energies during the April 1997 outburst (see also [21,24]). Detailed analysis indeed suggests that one-component SSC models cannot fit both the April 1997 SEDs and the lightcurves from X-ray to TeV, and that (at least) an additional, moderately variable low energy component contributing in the energy range between $3-25 \mathrm{keV}$ is required [33,41. A similar conclusion seems to hold for the strong X-ray outburst observed in July 1997 [34]. Interestingly, observations in 1998 also provide evidence for an additional component in the optical regime, possible associated with the SS disk component in our hybrid disk model (see also [25] for an alternative interpretation): As shown by Massaro et al. [41] optical to X-ray data taken in June 1998 indicate that the optical spectrum is steep and does not match the low energy extrapolation of the X-ray spectrum, hence suggesting the presence of different emission components in the optical and in the X-ray regime as naturally expected in our model.

Monitors in both the X-rays and the TeV emission show evidence for a 23day periodicity during the 1997 high state. As demonstrated above the 23-day period in the X-ray light curve may be caused by a pulsational instability in two-temperature accretion disk, and via the inverse Compton process result in the same periodicity in the $\mathrm{TeV}$ light-curve. A pulsational instability occurring in a two-temperature disk with transition radius $r_{0} \sim(48-65) r_{g}$ will result in a recurrence timescale of 8 to 100 days.

Based on the observed $\mathrm{TeV}$ variability we have employed a characteristic black hole mass of $9 \times 10^{7} M_{\odot}$ in our calculations. We note that quite different central mass estimates for Mkn 501 have been claimed in the literature, ranging from several times $10^{7}$ (mainly based on high energy emission properties) up to $10^{9} M_{\odot}$ (based on host galaxy observations), see e.g. [7, 12, 18, 19,54]. However, as shown by Rieger \& Mannheim [54] uncertainties associated with host galaxy observations may easily lead to an overestimate of the central black hole mass in Mkn 501 by a factor of three and thus reduce the implied central mass to $\simeq(2-3) \times 10^{8} M_{\odot}$, a value in fact recently confirmed by an independent analysis of central mass constraints derived from host galaxy observations [68]. Moreover, as argued by the same authors some of the apparent disagreement in central mass estimates may possibly be resolved if a binary black hole system exists in the center of Mkn 501, see also [53,54,65], similar as in the case of OJ 287 [60]. For example, if Mkn 501 harbours a binary system with a 
more massive primary black hole of $\lesssim 10^{9} M_{\odot}$ and a less massive (jet-emitting) secondary black hole of $\sim 10^{8} M_{\odot}$, the mass ratio $\rho=\mathrm{m} / M$ would be of order 0.1 , which may compare well with the result $\rho<0.25$ estimated for OJ 287 [38. While our characteristic black hole mass employed falls well within the above noted range, we note that the SLE pulsational instability model may still work successfully, if a higher black hole mass is used. For example, if one adopts $M=3 \times 10^{8} M_{\odot}$ and $P=23$ days, one obtains $\alpha \leq 0.07, r_{0 *}=46$ and $\dot{M} \simeq 0.008 \dot{M}_{\mathrm{Edd}}$.

Our analysis is based on a specific disk model (SLE) which is open to questions, in particular with respect to its possible stability properties. We note however, that a relatively small change in the usually employed viscosity description may already lead to a thermally stable configuration [49]. On the other hand, it may as well be possible that the SLE configuration represents a quasi-transient phenomenon associated with those changes in accretion history that probably initiate the high states. An alternative (inner) disk configuration of interest may be represented by an optically thin two-temperature ADAF solution 44,71. Such a configuration can exist for accretion rates $\dot{m}=\dot{M} / \dot{M}_{\text {Edd }}$ below a critical rate $\dot{m}_{\text {crit }} \simeq 0.3 \alpha^{2} \simeq 0.019$, where the canonical ADAF value of $\alpha=0.25$ has been employed [44]. ADAFs are generally less luminous than a standard disk, with the typical ADAF luminosity given by $L_{A} \simeq 0.02\left(\dot{m} / \alpha^{2}\right) \dot{M} c^{2}[71]$. Using the constraints above, the possible ADAF luminosity for Mkn 501 becomes $L_{A} \leq 1.3 \times 10^{43} \mathrm{erg} / \mathrm{s}$, which is already about an order of magnitude smaller than required by the X-ray analysis. This suggests that - at least during its high state - an optically thin ADAF is not a viable option for Mkn 501.

Based on Eq. (7) we can estimate the variation rate of the period due to the motion of the instability

$$
\delta P / P=\frac{3}{2} P \frac{1}{r} \frac{\partial r}{\partial t}
$$

Using $v_{r}=\frac{\partial r}{\partial t}, \dot{M}=4 \pi \rho h r v_{r}$ and Eqs. (4) and (5), one finds

$$
\delta P / P=0.44 \alpha^{-7 / 6} \dot{M}_{24}^{5 / 6} M_{7}^{-5 / 6} r_{*}^{-1 / 4} \zeta^{-1 / 6}
$$

For $\alpha=0.28$ the relevant parameters result in $\delta P / P=0.23$. From the period analysis performed by Kranich et al. [30] a $3 \sigma$ deviation in period corresponds to 6.67 days. If we take this deviation as the intrinsic variation on the periodicity $(\mathrm{P})$, then a $\delta P / P$ of $\frac{6.67}{23}=0.29$ can be estimated from the results by Kranich et al. [30]. As this estimate assumes that the deviation of the period is only affected by the motion of the instability, while it may in fact be caused by more than one effect, our theoretical $\delta P / P$ should not be greater than the 
observational results. We conclude that the observed 23-day QPOs in Mkn 501 might be caused by the instability of a two-temperature accretion disk. The model presented here may thus offers an alternative explanation to the binarydriven helical jet model of Rieger \& Mannheim (2000). Comprehensive computational modelling of the pulsational instability in a two-temperature, cooling dominated disk will be essential to verify this in more detail.

Our model predicts that a period correlation in the X-ray and $\gamma$-ray should always be present during an active source stage, while the period of the QPOs may vary as the instability region could change from one high state to the other.

\section{Acknowledgements}

We would like to thank Prof. K.S. Cheng, J.M. Wang, Y. Lu, L. Zhang and Dr. D. Kranich for useful discussions, and the anonymous referees for very useful comments that helped to improved the presentation. This work is partially supported by the National 973 project (NKBRSF G19990754), the National Science Fund for Distinguished Young Scholars (10125313), the National Natural Science Foundation of China (10573005, 10633010), the Fund for Top Scholars of Guangdong Province (Q02114) and a Cosmogrid Fellowship (FMR). We also acknowledge financial support from the Guangzhou Education Bureau and Guangzhou Science and Technology Bureau. 


\section{References}

[1] Aharonian F., Akhperjinian A.G., Barrio J.A., et al. 1999a, A\&A, 342, 69

[2] Aharonian F., Akhperjanian A.G., Barrio J.A. et al. 1999b, A\&A, 349, 11

[3] Albert J., et al. (MAGIC collaboration) 2007, ApJ in press (astro-ph/0702008)

[4] Bloom S., Marscher A.P. 1996, ApJ, 463, 555

[5] Blumenthal G.R., Yang L.T., Lin D.N.C. 1984, ApJ, 287, 774

[6] Buckley, J. H.; Akerlof, C. W.; Biller, S., et al. 1996, ApJ 472 L9

[7] Cao X. 2002, ApJ, 570, L13

[8] Cao X. 2003, ApJ, 599, 147

[9] Catanese M., Bradbury S.M., Breslin A.C., et al. 1997, ApJ 487, L143

[10] Catanese M., Weekes T.C. 1999, PASP, 111, 1193

[11] Chen X., Taam R.E. 1995, ApJ, 441, 354

[12] De Jager O.C., Kranich D., Lorentz E., Kestel M. 1999, Proc. 26th ICRC (Salt Lake City), 3, 346

[13] Dermer C.D., Schlickeiser R., Mastichiadis A. 1992, A\&A 256, L27

[14] Dermer C.D., Schlickeiser R. 1993, ApJ 416, 458

[15] Dermer C.D., Schlickeiser R. 1994, ApJS, 90, 945

[16] Dermer C.D., Sturner S.J., Schlickeiser R. 1997, ApJS, 109, 103

[17] Djannati-Atai A., Piron F., Barrau A., et al. 1999, A\&A, 350, 17

[18] Falomo R., Kotilainen J. K., Treves A. 2002, ApJL, 569, L35

[19] Fan J.H. 2005, A\&A, 436, 799

[20] Fan J.H., Lin R.G. 1999, ApJS, 121, 131

[21] Ghisellini G. 1998, in: The Active X-ray Sky: Results from BeppoSAX and RXTE, ed. by L. Scarsi et al., Amsterdam, 397

[22] Hayashida N., Hirasawa H., Ishikawa F., et al. 1998, ApJL, 504, L71

[23] Honma F., Matsumoto R., Kato S. 1992, PASJ, 44, 529

[24] Kataoka J., Mattox J.R., Quinn J., et al. 1999, ApJ, 514, 138

[25] Katarzynski K., Sol. H., Kus A. 2001, A\&A 367, 809

[26] Kato S. 1978, MNRAS, 185, 629 
[27] Kley W., Papaloizou J.C.B., Lin D.N.C. 1993, ApJ, 409, 739

[28] Konopelko A. 1999, APh, 11, 135

[29] Konopelko A., Mastichiadis A., Kirk, J., et al. 2003, ApJ 597, 851

[30] Kranich D., De Jager O.C., Kestel M., et al. 1999, Proc. 26th ICRC (Salt Lake City), 3, 358

[31] Kranich D., De Jager O.C., Kestel M., et al. 2001, Proc. 27th ICRC (Hamburg), 7,2631

[32] Krawczynski H., Coppi P.S., Maccarone T., Aharonian F.A. 2000, A\&A, 353, 97

[33] Krawczynski H., Coppi P.S., Aharonian F. 2002, MNRAS 336, 721

[34] Lamer G., Wagner S.J. 1998, A\&A, 331, L13

[35] Laor A. 2003, ApJ, 590, 86

[36] Lightman A.P. 1974, ApJ, 194, 419

[37] Lightman A.P., Eardley D. 1974, ApJ, 187, L1

[38] Liu F.K., Wu X-B. 2002, A\&A, 388, L48

[39] Macomb, D. J., Akerlof, C. W., Aller, H. D., et al. 1995, ApJ, 449, L99

[40] Manmoto T., Takeuchi M., Mineshige S., et al. 1996, ApJ, 464, L135

[41] Massaro E., Perri M., Giommi P., et al. 2004, A\&A, 422, 103

[42] Mastichiadis A., Kirk J.G. 1997, A\&A, 320, 19

[43] Milsom J.A., Taam R.E. 1997, MNRAS, 286, 358

[44] Narayan R., Mahadevan R., Quataert, E. 1998, in: Theory of Black Hole Accretion Disks, ed. by M.A. Abramowicz et al., p.148

[45] Osone S., 2006, APh, 26, 209

[46] Papaloizou J.C.B., Stanley G.Q.R. 1986, MNRAS, 220, 593

[47] Petry D., Böttcher M., Connaughton V., et al 2000, ApJ, 532, 742

[48] Pian E., Vacanti G., Tagliaferri G., et al. 1998, ApJ, 492, L17

[49] Piran T. 1978, ApJ, 221, 652

[50] Pringle J.E. 1976, MNRAS, 177, 65

[51] Protheroe R.J. et al. 1997, Proc. 25th ICRC (Durban), 8, 317

[52] Quinn J., Akerlof C.W., Biller S., et al. 1996, ApJ, 456, L83

[53] Rieger F.M. Mannheim K. 2000, A\&A, 359, 948 
[54] Rieger F.M. Mannheim K. 2003, A\&A, 397, 121

[55] Rieger F.M., Bosch-Ramon V., Duffy P. 2007, Ap\&SS 309, 119

[56] RXTE 1999, http://space.mit.edu/XTE/asmlc/ASM.html

[57] Sambruna R., Maraschi L., Urry C.M. 1996, ApJ, 474, 639

[58] Shapiro S.L., Lightman A.P., Eardley D.M. 1976, ApJ, 204, 187

[59] Shakura N.I., Sunyaev R.A. 1973, A\&A, 24, 337

[60] Sillanpää A., Haarala S., Valtonen M. J., et al. 1988, ApJ, 325, 628

[61] Stickel M., Fried J.W., Kühr H. 1993, A\&AS, 98, 393

[62] Tavecchio F., Maraschi L., \& Ghisellini G. 1998, ApJ, 509, 608

[63] Takahashi, T.; Tashiro, M.; Madejski, G. et al. 1996, ApJ, 470, L89

[64] Tavecchio F., Maraschi L., Pian E., et al. 2001, ApJ, 554, 725

[65] Villata M., Raiteri C. M. 1999, A\&A, 347, 30

[66] Wandel A., Liang E.P. 1991, ApJ, 380, 84

[67] Wandel A., Urry M.C. 1991, ApJ, 367, 78

[68] Woo, J-H., Urry, M.C., et al. 2005, ApJ, 631, 762

[69] Wu X.B. 1997, MNRAS, 292, 113

[70] Yang L.T., Henning T., Lu Y., Wu X.B. 1997, MNRAS, 288, 965

[71] Yi I. 1999, in: Astrophysical Disks, ed. by J.A. Sellwood \& J. Goodman, ASP Conf.Ser. 160, 279 